\title{
Suicide substrate reaction-diffusion equations: Varying the source
}

\author{
Meghan A. Burke† And P. K. Maini \\ Centre for Mathematical Biology, Mathematical Institute, \\ 24-29 St Giles', Oxford OX1 3LB, UK
}

\section{J. D. Murray}

Applied Mathematics Department FS-20, University of Washington, Seattle, Washington 98195 , USA

[Received 29 September 1992 and in revised form 2 April 1993]

\begin{abstract}
The suicide substrate reaction is a model for certain enzyme-inhibiting drugs. This reaction system is examined assuming that the substrate diffuses freely while the enzyme remains fixed. Two sets of initial and boundary conditions are examined: one modelling an instantaneous point source, akin to an injection of substrate, the other, a continuous point source, akin to a continuing influx, or intravenous drip, of substrate. The quasi-steady-state assumption is applied to obtain analytical solutions for a limited parameter space. Finally, further applications of numerical and analytical experimentation on pharmaceutical mechanisms are described.
\end{abstract}

Keywords: suicide substrate; reaction-diffusion equations; analytical solution; quasisteady-state assumption; instantaneous point source; continuous point source.

\section{Introduction}

This is the third in a sequence of papers generalizing enzyme-substrate analyses (Burke et al., 1990; Maini et al., 1991). The system we are most interested in is the 'suicide substrate' system, represented by

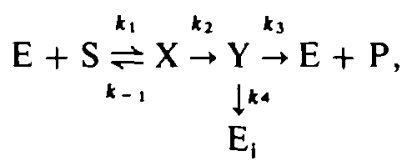

where $\mathrm{E}, \mathrm{S}$, and $\mathrm{P}$ denote enzyme, substrate, and product, respectively; $\mathrm{X}$ and $\mathrm{Y}$, enzyme-substrate intermediates; $E_{i}$, inactivated enzyme; and the $k$ 's, the positive rate constants. In this system, the enzyme reacts with the suicide substrate $S$ to form intermediate $\mathrm{Y}$, which can either convert to product and regenerate the unchanged enzyme or become irreversibly inactivated enzyme. In this way, a suicide substrate can specifically target an enzyme for inactivation. Furthermore, suicide substrates

† Present address: Pittsburgh Cancer Institute, Division of Basic Research, Biomedical Science Tower, DeSoto at O'Hara Street, Pittsburgh, Pennsylvania 15213, USA. 
are not harmful in their common form. Hence, they are very useful as drugs (Seiler et al., 1978; Walsh et al., 1984).

Using the law of mass action on the suicide substrate reaction, along with conservation of enzyme, we obtain a system of four rate equations:

$$
\begin{aligned}
{[\mathrm{S}]_{\mathrm{t}} } & =-k_{1}\left(E_{0}-[\mathrm{X}]-[\mathrm{Y}]-\left[\mathrm{E}_{\mathrm{i}}\right]\right)[\mathrm{S}]+k_{-1}[\mathrm{X}], \\
{[\mathrm{X}]_{\mathrm{t}} } & =k_{1}\left(E_{0}-[\mathrm{X}]-[\mathrm{Y}]-\left[\mathrm{E}_{1}\right]\right)[\mathrm{S}]-\left(k_{-1}+k_{2}\right)[\mathrm{X}], \\
{[\mathrm{Y}]_{\mathrm{t}} } & =k_{2}[\mathrm{X}]-\left(k_{3}+k_{4}\right)[\mathrm{Y}], \\
{\left[\mathrm{E}_{\mathrm{i}}\right]_{\mathrm{t}} } & =k_{4}[\mathrm{Y}]
\end{aligned}
$$

where [ ] denotes concentration. In a previous paper (Burke et al., 1990), we applied a new small parameter $\varepsilon$ to the above space-independent problem. The new $\varepsilon$ was defined by Segel \& Slemrod (1989) as

$$
\varepsilon=\frac{E_{0}}{S_{0}+K_{M}}
$$

where $E_{0}$ and $S_{0}$ are the initial concentrations of free enzyme and substrate, respectively, and $K_{\mathrm{M}}=\left(k_{-1}+k_{2}\right) / k_{1}$ is the Michaelis constant. This $\varepsilon$ was introduced as a more general small parameter than $\varepsilon_{\mathrm{h}}=E_{0} / S_{0}$, which was previously used by several authors (see e.g. Heineken et al., 1967, and the books by Murray, 1977, and Keener, 1988). By utilizing this new parameter, which is small when $K_{M}$ is large, even if $E_{0}$ and $S_{0}$ are of the same order of magnitude, it is possible to solve the system in two time regimes: when $t / \varepsilon$ is $O(1)$ and when $t$ is $O(1)$, that is, before and during the quasi-steady-state phase, when the enzyme is saturated with substrate and the quasi-steady-state assumption (QSSA) is valid. In this way, one can obtain a solution uniformly valid for all time.

In a second paper (Maini et al., 1991), we discussed the spatially varying problem by introducing injection and diffusion of the substrate. There, we find that the region where the QSSA holds is no longer a small region close to $t=0$, but varies from a small ring about the origin to a disc in space which expands in time, depending on the enzyme reaction. However, we do find the consistency that when

$$
\varepsilon(r, t)=\frac{\vec{E}}{\bar{S}+K_{M}}
$$

is small, where $\bar{E}$ and $\bar{S}$ are some typical values of enzyme and substrate, the QSSA is valid. This emphasizes the significance of the new $\varepsilon$, as it introduced $K_{\mathrm{M}}$, the only quantity which is constant in space and time.

In this paper, we examine more closely the case where the QSSA holds in the suicide substrate reaction, and attempt to solve the resulting system with both an instantaneous point source, representing a single injection of substrate, and a continuous point source, representing a continuous flow of substrate into the system.

\section{Reaction-diffusion system}

In enzyme reactions, the molecular weight of the enzyme is normally much larger than that of the substrate; in some cases it may be orders of magnitude larger. 
Furthermore, there are many instances where the enzyme is, in fact, physically bound to a membrane while the substrate diffuses freely. For these reasons, it is reasonable to model the spatial dependence in an enzyme-substrate reaction by diffusion of the substrate while the enzyme is held fixed.

It is notable that, in assuming $\mathrm{E}$ is fixed, we can assume that any complex involving $E\left(X, Y\right.$, and $\left.E_{l}\right)$ is also fixed. As a first step towards modelling spatial dependence, we assume that the medium is homogeneous; we ignore effects due to blood cells, cell membrane, or blood vessel barriers, and consider the diffusion to be modelled by Fick's law with constant diffusion coefficient.

The scenario we would like to examine is as follows. Initially, an amount of substrate is injected into the medium at the origin. The substrate diffuses into the medium and reacts with the enzyme. In looking at the suicide substrate reaction, we wish to analyse the parameters that affect the size of the region and the degree to which the enzyme is inactivated.

Therefore, in our simple case, we use a Dirac delta-function as the initial condition for [S], with $S_{0}$ as its coefficient. This implies that the total amount of substrate is $S_{0}$ at the origin and zero elsewhere at the beginning of the reaction (time $=0$ ). The free enzyme, on the other hand, is present in uniform concentration, $E_{0}$, throughout the medium. At the beginning of the reaction, there are no enzymesubstrate complexes.

The full three-dimensional version of the model is not amenable to analysis and can only be solved numerically. To gain insight into the behaviour of the system with diffusion, we therefore restrict ourselves to the special case of radial symmetry-this enables us to carry out a reasonably complete analysis and to understand the effects of diffusion. We represent the spatial dimension by $r$, distance from the origin, and use $\nabla^{2}$ to indicate the spatial Laplacian in $n$ dimensions. For illustrative purposes, we use $n=1$. The constant coefficient of diffusion is $D$.

To investigate the effects of diffusion we consider a large domain, so that boundary conditions play only a minimal role in the initial stages of the reaction. Since the problem is symmetric about $r=0$ for $n>1$, we have a no-flux condition at the origin. We also use a no-flux condition at the periphery, $r=L$, so that substrate does not leak out from the boundary. To ensure that this holds for $n=1$, we impose no-flux conditions at $r=0$ in this case also. With these conditions, we have, for the spatially dependent suicide substrate reaction,

$$
\begin{aligned}
{[\mathrm{S}]_{t} } & =-k_{1}\left(E_{0}-[\mathrm{X}]-[\mathrm{Y}]-\left[\mathrm{E}_{\mathrm{i}}\right]\right)[\mathrm{S}]+k_{-1}[\mathrm{X}]+D \nabla^{2}[\mathrm{~S}] \\
{[\mathrm{X}]_{t} } & =k_{1}\left(E_{0}-[\mathrm{X}]-[\mathrm{Y}]-\left[\mathrm{E}_{1}\right]\right)[\mathrm{S}]-\left(k_{-1}+k_{2}\right)[\mathrm{X}] \\
{[\mathrm{Y}]_{t} } & =k_{2}[\mathrm{X}]-\left(k_{3}+k_{4}\right)[\mathrm{Y}] \\
{\left[\mathrm{E}_{\mathrm{i}}\right]_{t} } & =k_{4}[\mathrm{Y}]
\end{aligned}
$$

with initial conditions

$$
\begin{gathered}
{[\mathrm{S}](0, r)=S_{0} \delta(r),} \\
{[\mathrm{X}](0, r)=[\mathrm{Y}](0, r)=\left[\mathrm{E}_{\mathrm{i}}\right](0, r)=0,}
\end{gathered}
$$


and boundary conditions

$$
\begin{aligned}
{[\mathrm{S}]_{r}(t, 0) } & =[\mathrm{S}]_{r}(\mathrm{t}, L)=0 \\
{[\mathrm{X}]_{r}(t, 0) } & =[\mathrm{X}]_{r}(t, L)=0, \\
{[\mathrm{Y}]_{r}(t, 0) } & =[\mathrm{Y}]_{r}(t, L)=0, \\
{\left[\mathrm{E}_{\mathrm{i}}\right]_{r}(t, 0) } & =\left[\mathrm{E}_{\mathrm{i}}\right]_{r}(t, L)=0,
\end{aligned}
$$

where we have used a Dirac delta-function initial condition to simulate a fixed amount $S_{0}$ of substrate injected initially at $r=0$. For all subsequent numerical simulations, we approximate the delta-function by a tent function.

\section{The quasi-steady-state assumption}

When $D=0$, it was found previously (Burke et al., 1990) that the quasi-steady-state assumption is valid if

$$
\varepsilon=\frac{E_{0}}{S_{0}+K_{M}}
$$

is small. Keeping this in mind for the $D \neq 0$ situation, intuitively we may say that where some related (but now variable) quantity

$$
\varepsilon(r, t)=\frac{\bar{E}}{\bar{S}+K_{M}}
$$

is small, the quasi-steady-state assumption holds. We introduce this new notation of $\varepsilon$ as a function of $r$ and $t$ to emphasize that it is no longer constant. Specifically, $\bar{S}$ is not constant. In fact, although we use $[S](0, r)=S_{0} \delta(r)$ as an initial condition, $S_{0}$ is not a good characteristic value of $\bar{S}$. We think of $\bar{S}$ intuitively as the typical amount of substrate that one observes at a fixed point in space. Although the delta-function initial condition implies that a space point near $r=0$ has zero initial concentration of substrate, very quickly a large amount of substrate diffuses towards that point. In contrast, a point far from $r=0$ also has zero initial concentration of substrate, but substrate only slowly enters its neighbourhood in very small amounts. Intuitively, we would like to say that these two points have different $\bar{S}$, but as yet we cannot quantify this.

Moreover, neither is $\bar{E}$ constant. Again observing from a fixed space point, we begin with $[\mathrm{E}]=E_{0}$. The concentration of enzyme then decreases as it reacts with substrate which has diffused into the area. Enzyme may not increase again significantly as substrate diffuses out of the area, because some of the enzyme does not return to its original form, but is left inactivated. So $\bar{E}$ also varies in time and space in some as yet unquantified way.

Clearly, adding diffusion has greatly increased the complexity of the problem, so we must choose special cases in which we can carry out an analysis. In the next section, we examine the effects of using the QSSA in the case where $K_{\mathrm{M}} \gg E_{0}, S_{0}$. We will see that the QSSA is largely valid in this case, because $\varepsilon(r, t)$ is small for most of the domain. 


\section{Instantaneous point source analysis}

When $K_{\mathrm{M}}$ is large compared with $E_{0}$ and $S_{0}$, then the quasi-steady-state approximation holds for all time and space domains, because $K_{\mathrm{M}}$ is independent of time and space, and $E_{0}$ and $S_{0}$ are the maximum values of $\bar{E}$ and $\bar{S}$, respectively. Thus, $\varepsilon(r, t)$ is bounded above by $E_{0} / K_{\mathrm{M}}$, and is always small. To test this hypothesis, we compare numerical solutions of the full system with the solutions of the quasi-steady-state system.

For this and all other simulations of parabolic systems of partial differential equations, we used NAG routine D03PGF. When only one parabolic PDE was solved, NAG routine D03PAF was used.

Using the quasi-steady-state assumption on (2)-(5), we set the time derivatives of $[\mathrm{X}]$ and $[\mathrm{Y}]$ in (3) and (4) equal to zero. Solving (4) for [Y] and substituting into (3), we have

$$
\left.\begin{array}{l}
{[\mathrm{X}]=\frac{[\mathrm{S}]\left(E_{0}-\left[\mathrm{E}_{\mathrm{i}}\right]\right)}{\left\{1+k_{2} /\left(k_{3}+k_{4}\right)\right\}[\mathrm{S}]+K_{\mathrm{M}}},} \\
{[\mathrm{Y}]=\frac{k_{2}}{k_{3}+k_{4}} \frac{[\mathrm{S}]\left(E_{0}-\left[\mathrm{E}_{i}\right]\right)}{\left\{1+k_{2} /\left(k_{3}+k_{4}\right)\right\}[\mathrm{S}]+K_{\mathrm{M}}}}
\end{array}\right\}
$$

By adding equation (2) to equation (3) with the left-hand side equated to zero we obtain a simple equation for $[\mathrm{S}]_{\mathrm{t}}$ in terms of $[\mathrm{X}]$ :

$$
[\mathrm{S}]_{t}=-k_{2}[\mathrm{X}]+D \nabla^{2}[\mathrm{~S}]
$$

Substituting (13) into (14) and (5), we obtain a system of two equations for [S] and $\left[E_{1}\right]$,

$$
\begin{aligned}
{[\mathrm{S}]_{t} } & =\frac{-k_{2}[\mathrm{~S}]\left(E_{0}-\left[\mathrm{E}_{\mathrm{i}}\right]\right)}{\left\{1+k_{2} /\left(k_{3}+k_{4}\right)\right\}[\mathrm{S}]+K_{\mathrm{M}}}+D \nabla^{2}[\mathrm{~S}], \\
{\left[\mathrm{E}_{\mathrm{i}}\right]_{t} } & =\frac{k_{2} k_{4}}{k_{3}+k_{4}} \frac{[\mathrm{S}]\left(E_{0}-\left[\mathrm{E}_{\mathrm{i}}\right]\right)}{\left\{1+k_{2} /\left(k_{3}+k_{4}\right)\right\}[\mathrm{S}]+K_{\mathrm{M}}},
\end{aligned}
$$

as the quasi-steady-state equations.

Figure 1 compares the $[S]$ and $\left[E_{i}\right]$ solution surfaces of the full system (2)-(5) with the solution surfaces of the quasi-steady-state approximation (15)-(16). We call this the 'difference surface': it is determined by taking the magnitude of the difference of the two concentration values at each point, and dividing by their average. Thus, where the difference surface is zero, the quasi-steady-state assumption is valid. This picture shows that $K_{M}$ large ensures validity of the QSSA almost everywhere, except near the very beginning of the reaction. We therefore take (15) to be a valid approximation for [S] of the full system. To solve this equation we must examine the magnitude of the various terms.

Large $K_{\mathrm{M}}$ implies that $k_{-1}+k_{2} \gg k_{1}$. In practice, it is often true that the enzyme-binding step (the uptake of substrate) is the rate-limiting step, which implies that $k_{-1} \gg k_{1}$. So let us assume that $k_{1}$ and $k_{2}$ are $O(1)$, and $k_{-1}$ is $O(1 / \varepsilon)$. 

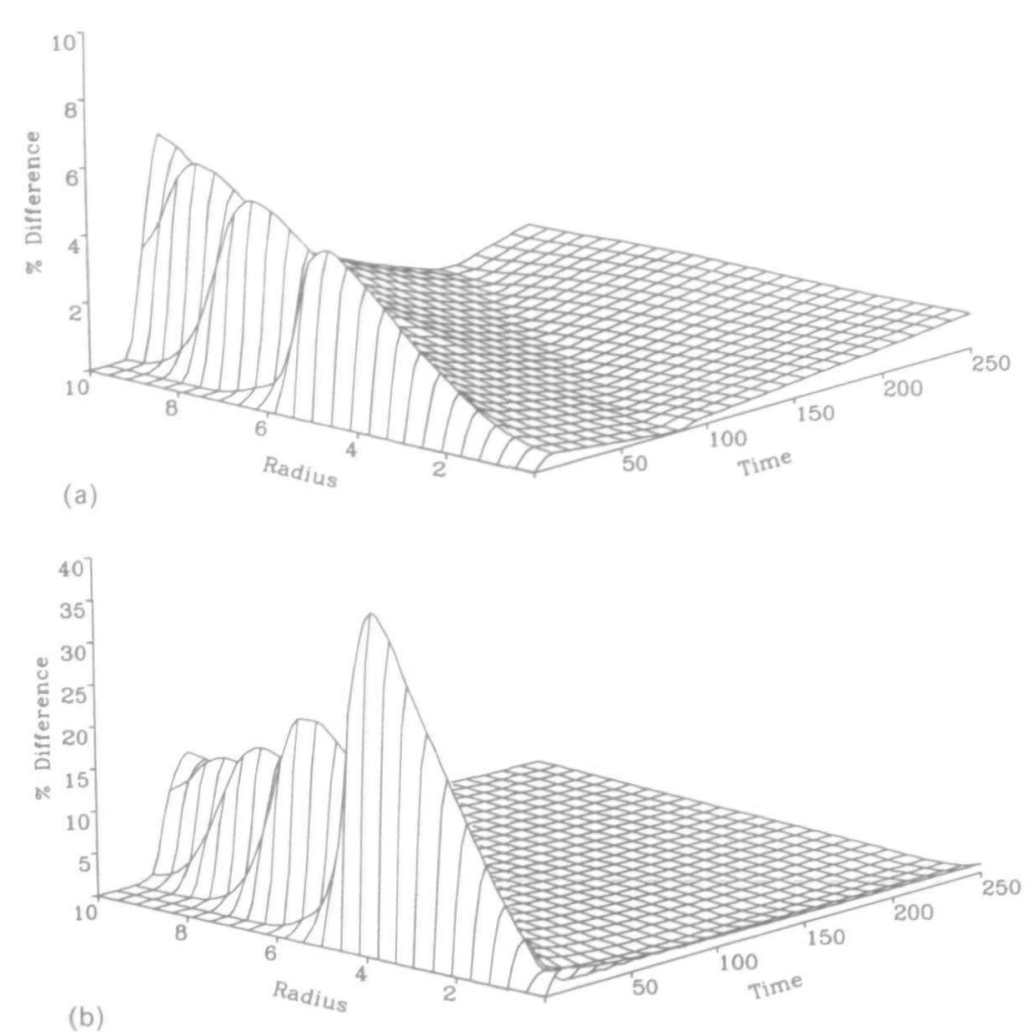

FiG. 1. Difference surface for (a) [S] and (b) [ $\left.E_{1}\right]$ from equations (2)-(5) and equations (15)-(16) (see text). Parameters: $k_{1}=0.05, k_{-1}=1.0, k_{2}=1.0, k_{3}=1.0, k_{4}=1.0, E_{0}=1.0, S_{0}=4.0, D=0.05$. With these parameter values, $K_{M}=40$. Note that away from zero for large $t$ there is a large percentage difference. This is because $[\mathbf{S}]$ is very small here, so that the denominator in the difference surface calculation tends to zero.

In (15) and (16), we notice that both denominators contain the term

$$
\left(1+\frac{k_{2}}{k_{3}+k_{4}}\right)[\mathrm{S}]+K_{\mathrm{M}} \text {. }
$$

Assuming that all the $k$ 's except $k_{-1}$ are $O(1)$, we may approximate (15) and (16) by

$$
\begin{aligned}
{[\mathrm{S}]_{t} } & =-\frac{k_{2}}{K_{\mathrm{M}}}[\mathrm{S}]\left(E_{0}-\left[\mathrm{E}_{\mathrm{i}}\right]\right)+D \nabla^{2}[\mathrm{~S}], \\
{\left[\mathrm{E}_{1}\right]_{\mathrm{t}} } & =\frac{k_{2} k_{4}}{\left(k_{3}+k_{4}\right) K_{\mathrm{M}}}[\mathrm{S}]\left(E_{0}-\left[\mathrm{E}_{\mathrm{i}}\right]\right),
\end{aligned}
$$

where we have used the observation that $K_{\mathrm{M}} \gg S_{0} \Rightarrow K_{\mathrm{M}} \gg[\mathrm{S}]$. That this is a good approximation to the quasi-steady-state equations (15)-(16) can be seen by solving both sets of equations numerically and calculating the difference surface (shown in Fig. 2). 


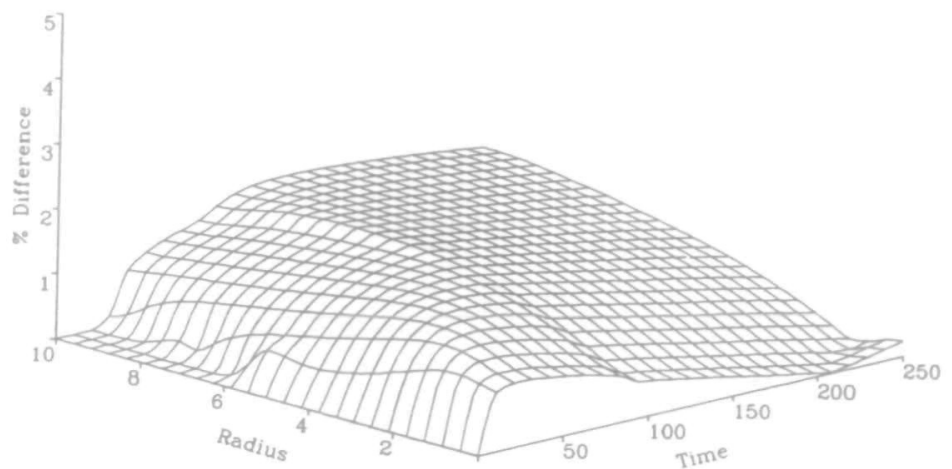

(a)

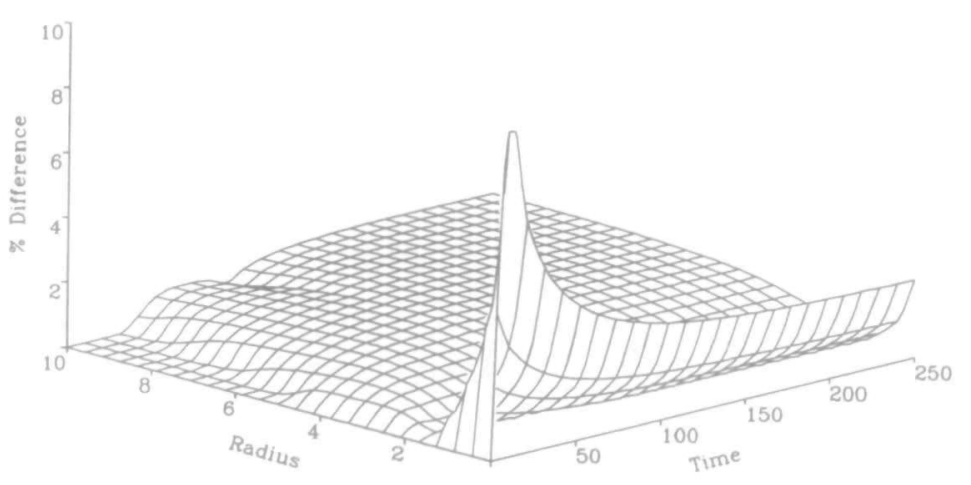

(b)

Fig. 2. Difference surface for (a) [S] and (b) $\left[E_{1}\right]$ from equations (15)-(16) and equations (18)-(19). Parameters: $k_{1}=0.05, k_{-1}=1.0, k_{2}=1.0, k_{3}=1.0, k_{4}=1.0, E_{0}=1.0, S_{0}=4.0, D=0.05$.

To analyse this system further, we first make the following substitution:

$$
A \equiv E_{0}-\left[\mathrm{E}_{1}\right] \text {. }
$$

The variable $A$ is the concentration of 'active' enzyme (that is, either free or in complex as $\mathrm{X}$ or $\mathrm{Y}$ ) and is easier to determine experimentally than the amount of inactive enzyme. The system (18)-(19) now becomes

$$
\begin{aligned}
{[\mathrm{S}]_{t} } & =-\frac{k_{2}}{K_{\mathrm{M}}}[\mathrm{S}] A+D \nabla^{2}[\mathrm{~S}], \\
A_{t} & =-\frac{k_{2} k_{4}}{\left(k_{3}+k_{4}\right) K_{\mathrm{M}}}[\mathrm{S}] A,
\end{aligned}
$$

which may be reduced to a single reaction-diffusion equation as follows (Britton, 1991).

Solving (22) for [S], we find

$$
[\mathrm{S}]=-\frac{A_{t}}{A} \frac{\left(k_{3}+k_{4}\right) K_{\mathrm{M}}}{k_{2} k_{4}} .
$$


Now introducing the function $W$, where

$$
W(r, t)=\int_{0}^{t}[\mathrm{~S}](r, \tau) \mathrm{d} \tau,
$$

we have

$$
\begin{aligned}
W(r, t) & =-\frac{\left(k_{3}+k_{4}\right) K_{M}}{k_{2} k_{4}} \int_{0}^{t} \frac{A_{t}}{A} \mathrm{~d} \tau \\
& =-\frac{\left(k_{3}+k_{4}\right) K_{\mathrm{M}}}{k_{2} k_{4}} \int_{A(r, 0)}^{A(r, t)} \frac{1}{\varphi} \mathrm{d} \varphi \\
& =-\frac{\left(k_{3}+k_{4}\right) K_{\mathrm{M}}}{k_{2} k_{4}} \ln \left(\frac{A(r, t)}{E_{0}}\right) .
\end{aligned}
$$

Solving (25) for $A$, we obtain

$$
A(r, t)=E_{0} \exp \left(-\frac{k_{2} k_{4}}{\left(k_{3}+k_{4}\right) K_{\mathrm{M}}} W(r, t)\right) .
$$

Now we integrate (21) with respect to $t$ and writing in terms of $W$, using (24), we get

$$
W_{t}=[\mathrm{S}](r, 0)-\frac{k_{2}}{K_{\mathrm{M}}} \int_{0}^{t}[\mathrm{~S}] A \mathrm{~d} \tau+D \nabla^{2} W .
$$

Substituting (23) and (26) into (27) leads, after some manipulation, to a single equation involving only $W$,

$$
W_{t}(r, t)=[\mathrm{S}](r, 0)+\frac{k_{3}+k_{4}}{k_{4}} E_{0}\left[\exp \left(-\frac{k_{2} k_{4}}{\left(k_{3}+k_{4}\right) K_{M}} W\right)-1\right]+D \nabla^{2} W,
$$

from which we can obtain [S], and hence an analytical expression for $A$, namely (26).

For $K_{M}$ large in comparison with $W$, it is reasonable to approximate

$$
\exp \left(-\frac{k_{2} k_{4}}{\left(k_{3}+k_{4}\right) K_{M}} W\right)
$$

by

$$
1-\frac{k_{2} k_{4}}{\left(k_{3}+k_{4}\right) K_{M}} W
$$

with the obvious limitation of positivity. This reduces (28) to

$$
W_{t}(r, t)=[\mathrm{S}](r, 0)-E_{0} \frac{k_{2}}{K_{\mathrm{M}}} W+D \nabla^{2} W,
$$

the solution to which can be found, for example, by Fourier transforms for the 
one-dimensional case. Thus

$$
\begin{aligned}
W(r, t)=\frac{S_{0}}{4 \sqrt{K D}}\left\{\exp \left(-\sqrt{\frac{K}{D}} r\right)\right. & {\left[1-\operatorname{erf}\left(-\sqrt{K t}+\frac{r}{2 \sqrt{D t}}\right)\right] } \\
+ & \left.\exp \left(\sqrt{\frac{K}{D}} r\right)\left[1-\operatorname{erf}\left(\sqrt{K t}+\frac{r}{2 \sqrt{D t}}\right)\right]\right\},
\end{aligned}
$$

where $K=\left(E_{0} k_{2}\right) / K_{\mathrm{M}}$.

We now obtain [S] by differentiating $W$ with respect to time:

$$
[\mathrm{S}]=\frac{S_{0}}{2 \sqrt{\pi D t}} \exp \left(-K t-\frac{r^{2}}{4 D t}\right) .
$$

This is, in fact, precisely the result obtained in the Michaelis-Menten case (see Maini et al., 1991). So, when (30) is a valid approximation of (29), that is, when $K_{M}$ is large compared with $W$, the mechanism of the suicide substrate reaction has little effect on the dynamics of $[\mathrm{S}]$.

This approximation is not simply a degeneration to a previous case. The analytical solution for $W$ in (32) can now be used to give an analytical solution for $A$ from (26). We thus have

$$
\begin{aligned}
A=E_{0} \exp \left(-\frac{k_{2} k_{4}}{\left(k_{3}+k_{4}\right) K_{M}} \frac{S_{0}}{4 \sqrt{K D}}\left\{\exp \left(-\sqrt{\frac{K}{D}} r\right)\left[1-\operatorname{erf}\left(-\sqrt{K t}+\frac{r}{2 \sqrt{D t}}\right)\right]\right.\right. \\
\left.\left.+\exp \left(\sqrt{\frac{K}{D}} r\right)\left[1-\operatorname{erf}\left(\sqrt{K t}+\frac{r}{2 \sqrt{D t}}\right)\right]\right\}\right)
\end{aligned}
$$

This analytical solution is only valid where (29) can be approximated by (30), that is, where $K_{\mathrm{M}}$ is large compared with $W$. Note that $K_{\mathrm{M}}$ is a straightforward combination of rate constants, but $W$ is more difficult to quantify intuitively. For a given $t$ and $r, W$ is the 'history' of [S] from the initial time point to time $=t$ at space point $r$. Thus several parameters have an effect on $W$. For example, when $D$ is large, $S$ diffuses quickly, and at a given space point, $W$ will be smaller than it would be if large amounts of $\mathrm{S}$ remained in the area (small $D$ ). Figure 3 shows the validity of the approximation for various values of $K_{\mathrm{M}}$ and $D$ for $[\mathrm{S}]$ and $\left[\mathrm{E}_{\mathrm{l}}\right]$. Notice that the approximation for [S] becomes worse than the approximation for [ $\left.\mathrm{E}_{\mathrm{i}}\right]$, and that $K_{M}$ appears to have a more pronounced effect than $D$, but that with increasing time (and hence increasing $W$ ) the approximation breaks down. Thus care is required when using (33) and (34), as the parameter space where they are valid is tight. However, these analytical solutions, expecially $(34)$ for $\left[E_{i}\right]$, can be informative in the parameter spaces where they are valid.

\section{Continuous source}

Previously, we considered the initial condition for substrate in our system to be a Dirac delta-function or a tent function approximation to it. This was intended to 


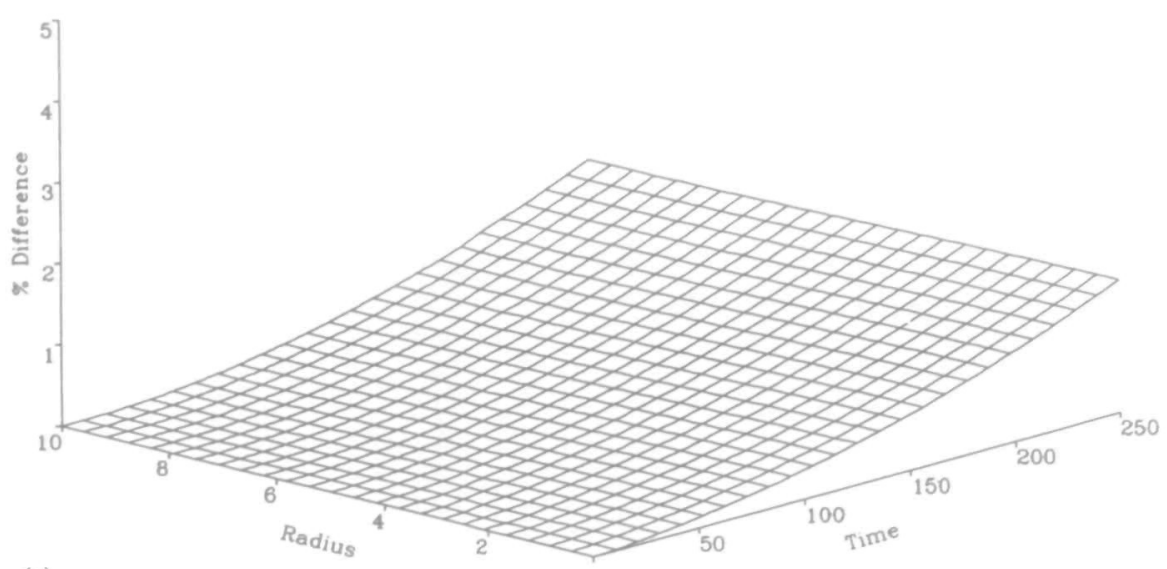

(a)

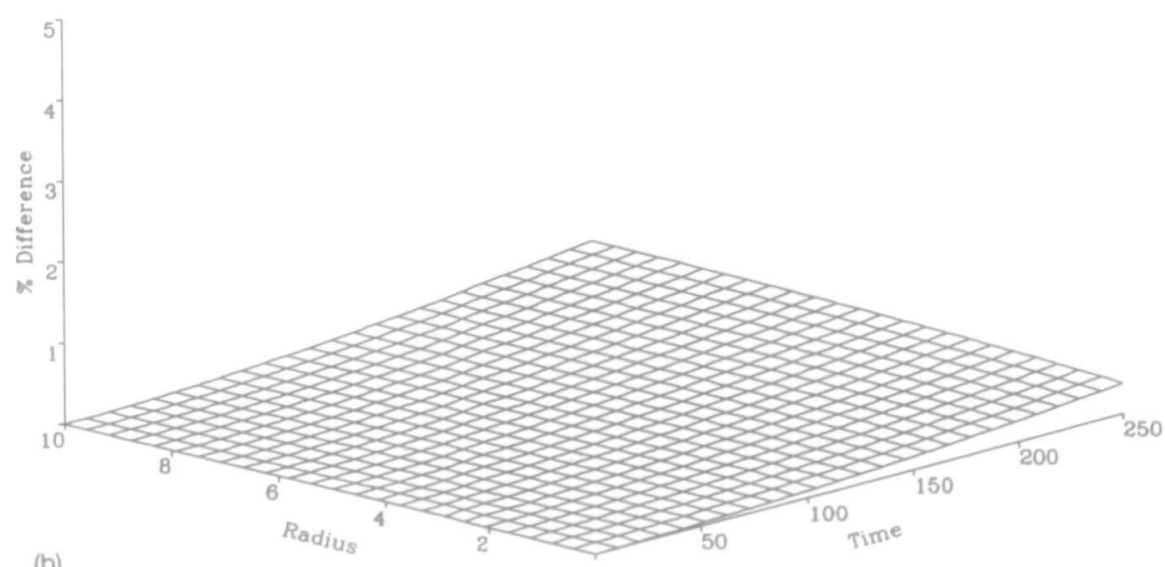

FIG. 3. Difference surfaces for $[S]$ and $\left[E_{l}\right]$ from the analytically solvable equations, (18) and (19) compared with [S] and $\left[E_{N}\right]$ from the full system $(2)-(5)$ for varnous values of $K_{M}$ and $D$. (a) [S] with $\mathrm{D}=0.5, k_{1}=0.005\left(K_{\mathrm{M}}=400.0\right)$. (b) $\left[\mathrm{E}_{\mathrm{f}}\right]$ with $D$ and $k_{1}$ as in (a).

represent an injection of substrate at the origin. We now consider the case of a continuous point source of substrate at the origin; that is, substrate flows into the system at a constant rate at that one point, much like an intravenous 'drip'.

The suicide substrate reaction is especially interesting under such conditions, as we can examine the dynamics of the inactivated enzyme, and draw preliminary medical conclusions from our study.

To represent a continuous source, we return to the differential equations (2)-(5) and change the boundary conditions from no flux at the origin to include influx of substrate at the origin. Thus the suicide substrate equations $(2)-(5)$ remain the same, 

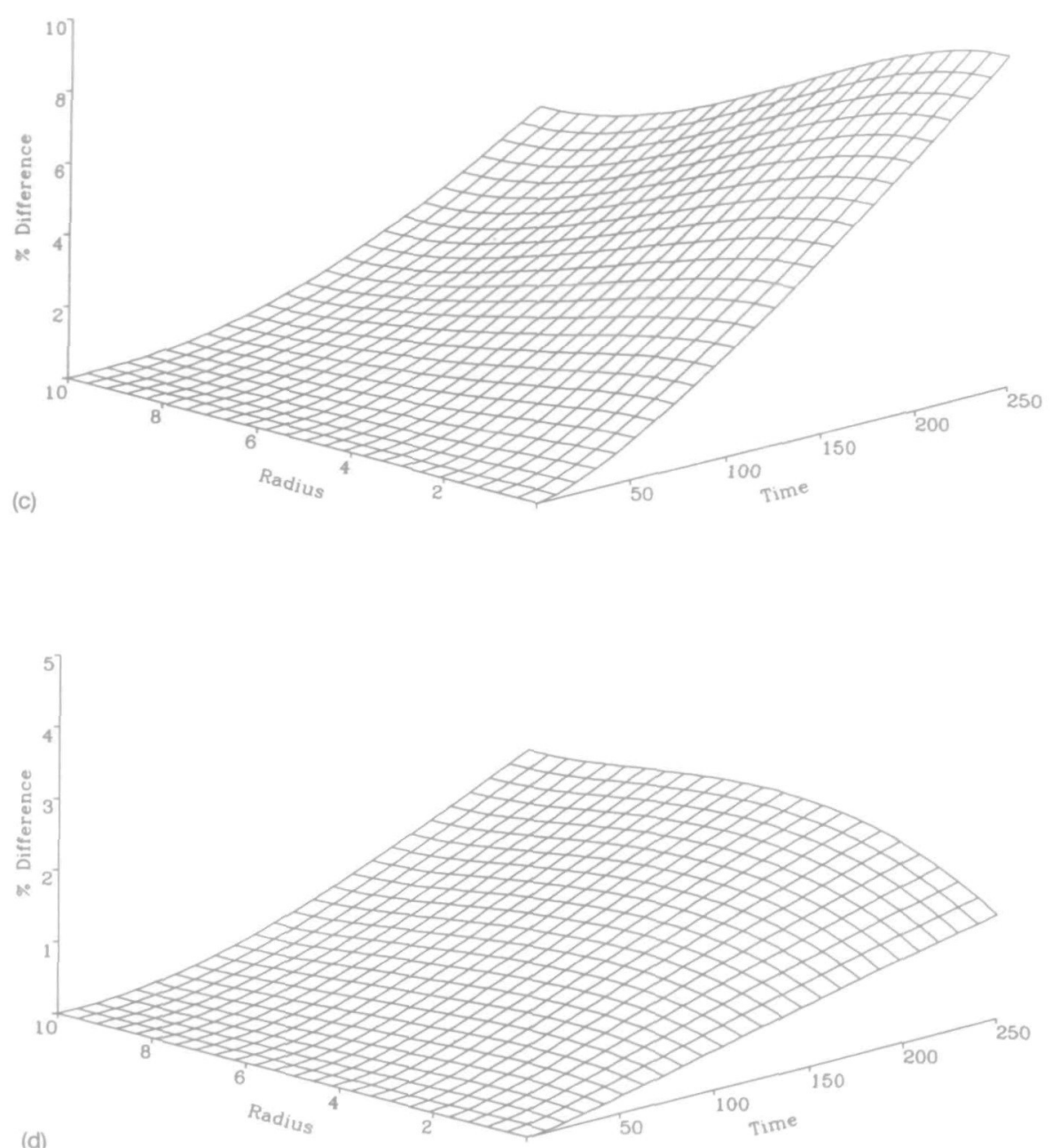

FiG 3. (Continued). (c) $[\mathrm{S}]$ with $D=0.1, k_{1}=0.01\left(K_{\mathrm{M}}=200.0\right)$. (d) $\left[\mathrm{E}_{1}\right]$ with $D$ and $k_{1}$ as in (c).

(continued)

but the initial and boundary conditions now become

$$
\left.\begin{array}{rrr}
{[\mathrm{S}](r, 0)=[\mathrm{X}](r, 0)=[\mathrm{Y}](r, 0)=\left[\mathrm{E}_{\mathrm{i}}\right](r, 0)=0,} \\
{[\mathrm{~S}]_{r}(0, t)=-Q,} & {[\mathrm{~S}]_{r}(L, t)=0,} \\
{[\mathrm{X}]_{r}(0, t)=0,} & {[\mathrm{X}]_{r}(L, t)=0,} \\
{[\mathrm{Y}]_{r}(0, t)=0,} & {[\mathrm{Y}]_{r}(L, t)=0,} \\
{\left[\mathrm{E}_{\mathrm{i}}\right]_{r}(0, t)=0,} & {\left[\mathrm{E}_{\mathrm{i}}\right]_{r}(L, t)=0,}
\end{array}\right\}
$$



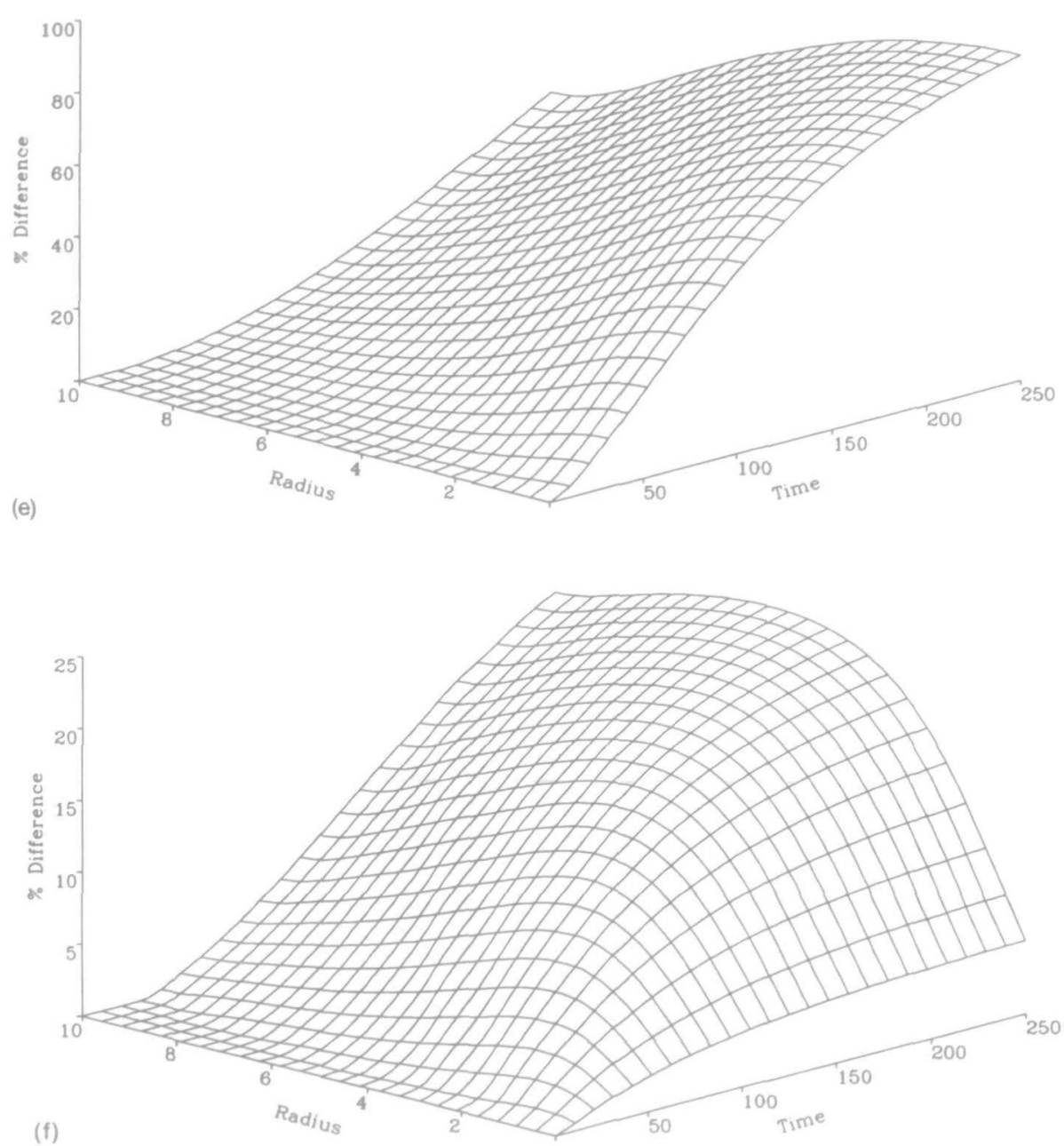

Fig. 3. (Continued). (e) [S] $D=0.05, k_{1}=0.05\left(K_{M}=40.0\right)$. (f) $\left[E_{1}\right]$ with $D$ and $k_{1}$ as in (e). Other parameters: $k_{-1}=1.0, k_{2}=1.0, k_{3}=1.0, k_{4}=1.0, E_{0}=1.0, S_{0}=4.0$.

where $-Q$ is the gradient of $[\mathrm{S}]$ at the origin (after $t=0$ ). By Fick's law, the flux

$$
F=-D \frac{\partial[\mathrm{S}]}{\partial r},
$$

so the first term in the first of (36) represents flux of $\mathrm{S}$ into the system at the origin with a flow rate $F=D Q$. We use (2)-(5) with (35) and (36) as a standard system for all numerical simulations of the suicide substrate continuous source system.

In the case of the continuous source, the QSSA should hold at a space point once 

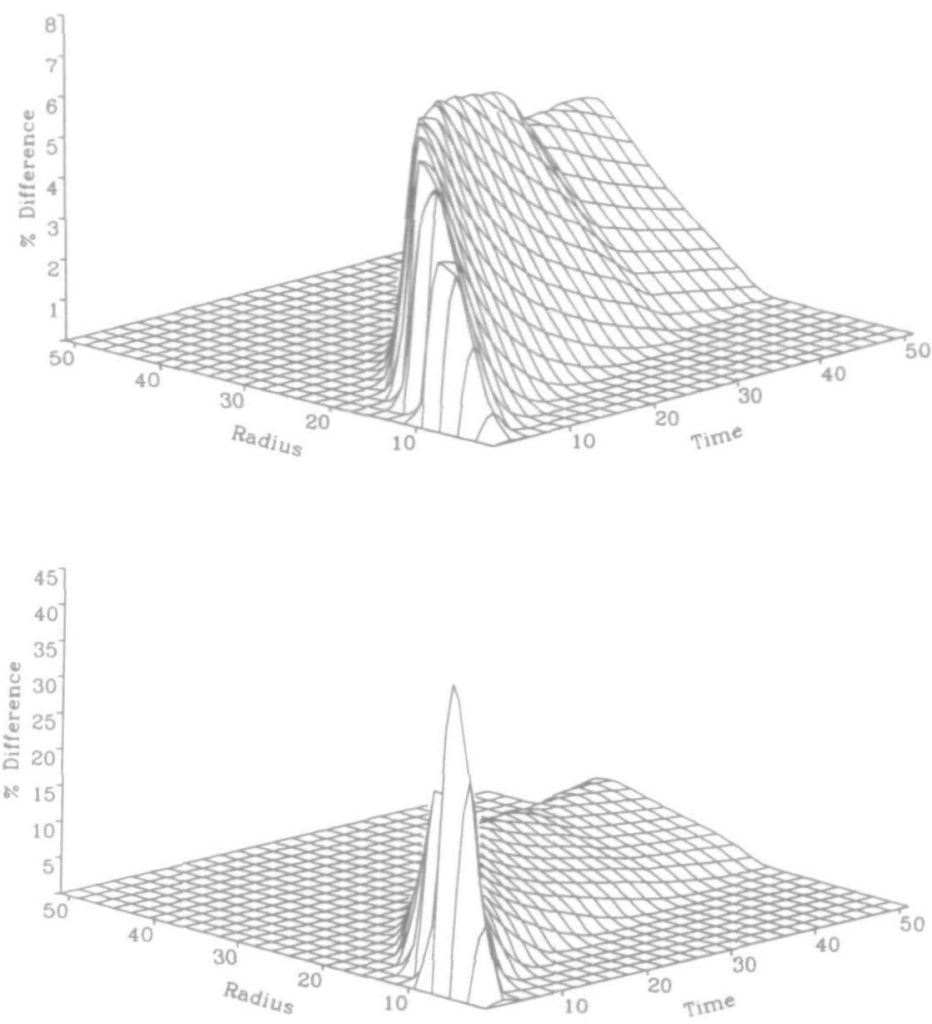

FIG. 4. Difference surface for (a) [S] and (b) [ $\left.\mathrm{E}_{\mathrm{i}}\right]$ of the full system (2)-(5) and of the QSS system (15)-(16) with large $K_{M}$ and continuous source conditions, (35) and (36). Parameters: $k_{1}=0.05, k_{-1}=1.0, k_{2}=1.0$, $k_{3}=1.0, k_{4}=1.0, E_{0}=1.0, Q=400.0, D=0.005$.

enough substrate has arrived to saturate the enzyme. If $K_{M}$ is large, this presaturation time period should be very short, and the QSSA will be a good approximation throughout the space-time domain. This implies that we can use (15) and (16) with the initial and boundary conditions describing a continuous source, namely (35) and (36). Figure 4 shows the difference surfaces for $[S]$ and $\left[E_{i}\right]$ of the full system (2)-(5) and of the QSS system (15)-(16) with large $K_{\mathrm{M}}$ and continuous source boundary conditions. With $\left[E_{i}\right]$ the difference is small everywhere except near the origin at very small time, while with [S] the difference is small (less than $10 \%$ ) everywhere. It is clear from these figures that we are justified in using the QSSA when $K_{\mathrm{M}}$ is large.

The solution for a problem with a continuous source may be obtained from the corresponding instantaneous source solution by integrating with respect to time (see e.g. Crank, 1975: p. 31). We apply this technique to the appropriate approximate solutions obtained earlier. There we found equation (33) as an approximate solution to the QSS system when $K_{\mathrm{M}}$ is large and $S_{0}$ is the coefficient of the delta-function point source. Now we can find the solution to the system with $F$ as the continuous 
source flow rate by integrating (33) with respect to time:

$$
\begin{aligned}
& {[S](r, t)=\frac{F}{4 \sqrt{K D}}\left\{\exp \left(-\sqrt{\frac{K}{D}} r\right)\left[1-\operatorname{erf}\left(-\sqrt{K t}+\frac{r}{2 \sqrt{D t}}\right)\right]\right.} \\
&\left.+\exp \left(\sqrt{\frac{K}{D}} r\right)\left[1-\operatorname{erf}\left(\sqrt{K t}+\frac{r}{2 \sqrt{D t}}\right)\right]\right\} .
\end{aligned}
$$

This is, in fact, $W$ from (32). As the boundary conditions and initial conditions for $\left[E_{i}\right]$ are the same whether we have an instantaneous source or a continuous source, we can still use the expression obtained for $\left[E_{i}\right]$ from (26):

$$
\left[\mathrm{E}_{\mathrm{i}}\right](r, t)=E_{0}-E_{0} \exp \left(-\frac{k_{2} k_{4}}{\left(k_{3}+k_{4}\right) K_{\mathrm{M}}} \int_{0}^{t}[\mathrm{~S}](r, \tau) \mathrm{d} \tau\right) .
$$

The expression for [S] in (38) cannot be integrated in closed form, but can be numerically integrated to give a solution for $\left[E_{i}\right]$.

We now determine the regions in parameter space wherein the expressions (38) and (39) found in the previous section via the QSSA are in close agreement with the solutions obtained by numerical simulation of the full system (2)-(5).

Figure $5(\mathrm{a}, \mathrm{b})$ shows the approximations $(38)$ and $(39)$ for $[\mathrm{S}]$ and $\left[\mathrm{E}_{3}\right]$ compared with the full system. In this figure, large $K_{\mathrm{M}}$ was used $\left(K_{\mathrm{M}}=4.0\right)$ with small $Q$ $(Q=0.04)$ so that the accumulation of $\mathrm{S}$, which is defined as $W$, remains small, making (30) a valid approximation of (29). One can see that the approximate solutions are in very close agreement with the full system. Figure $5(c, d)$ shows the same solutions, but with different parameters. Here $Q$ is larger $(Q=0.4)$, while $K_{\mathrm{M}}$ is kept at 4.0. These parameters are outside the domain specified when we obtained (38) and (39), so we expect to lose some accuracy. If we violate the restrictions further, and use smaller $K_{M}\left(K_{M}=0.8\right)$, we can see (Fig. $5(e, f)$ ) that the accuracy of our approximations deteriorates further. However, we now know that our approximations are valid in the specified parameter space, namely $K_{\mathrm{M}}$ large and $Q$ small.

\section{Discussion}

We have generalized the quasi-steady-state assumption to apply to the case where the diffusion coefficient $D \neq 0$ for two different boundary conditions. We have shown how the careful choice of a general small parameter $\varepsilon$ enables us to analyse certain types of reaction which could not be analysed using the classical small parameter. Specifically, we obtained solutions for an enzyme-suicide substrate reaction with an instantaneous point source of substrate.

We then used these analytical solutions to the instantaneous point source problem to obtain solutions to the continuous point source problem, with boundary conditions (35) and (36). For both sets of boundary conditions we have determined the regions in parameter space where our analytical solutions are valid.

Now that we have an idea of the suicide substrate reaction dynamics with a continuous source, a natural extension of this work is to examine the system with a switch-function source. That is, we would like to turn the source on and off, and 

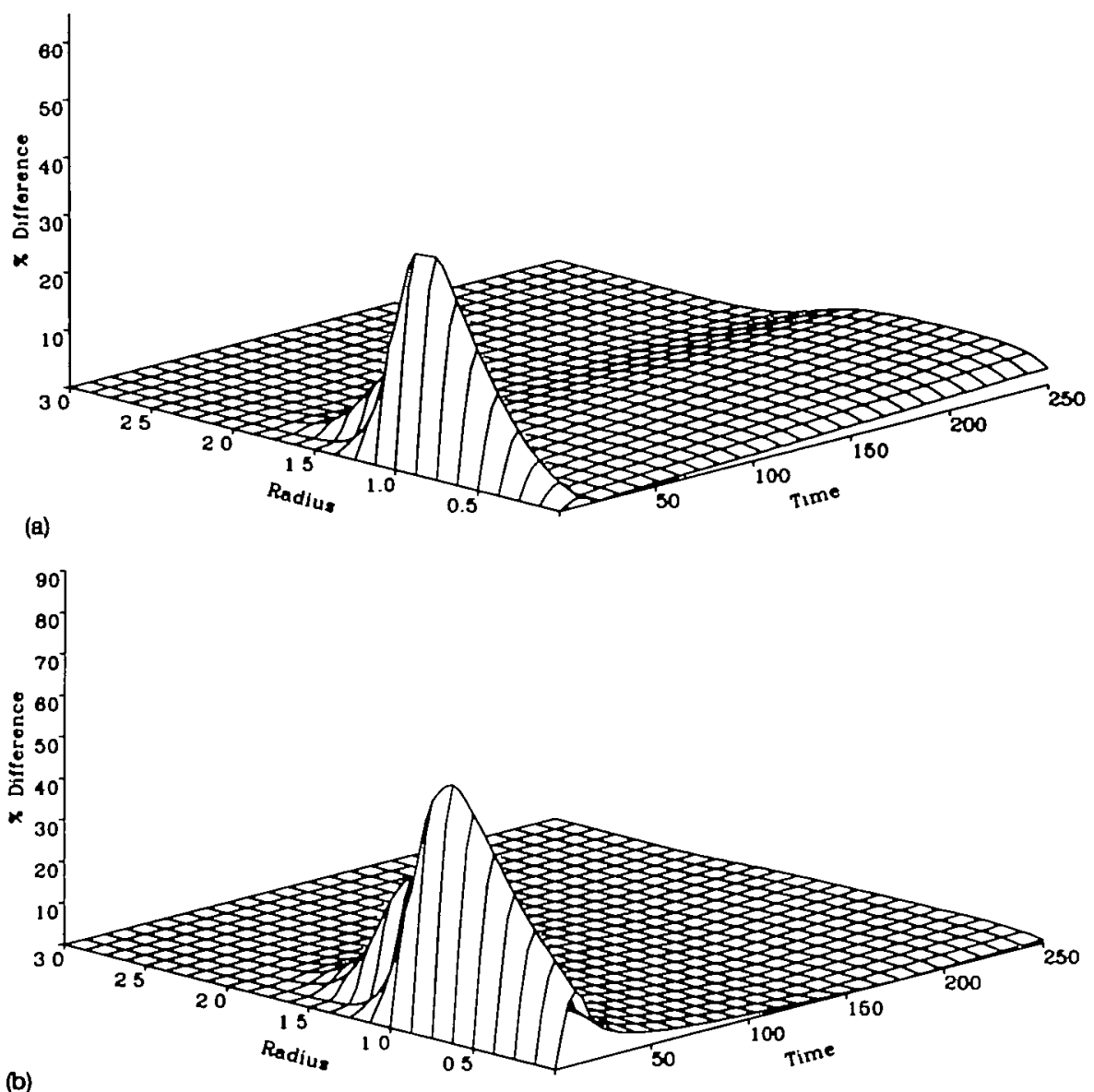

(b)

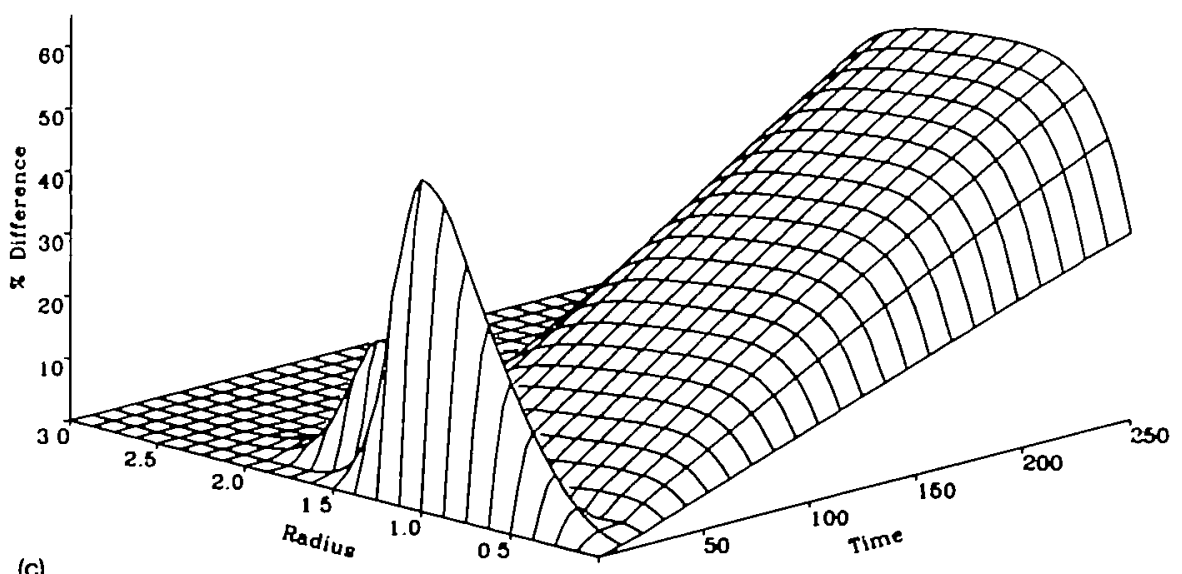

FiG. 5. Approximations for [S] and $\left[E_{1}\right],(38)$ and (39), compared with the numerical solutions of the full system (2)-(5). (a) and (b): [S] and $\left[\mathrm{E}_{1}\right]$ with $k_{1}=0.5, k_{-1}=1.0, k_{2}=1.0, Q=0.04$. (c): [S] with $k_{1}=0.5$, $k_{-1}=1.0, k_{2}=1.0, Q=2 \times 10^{-3}$. 


$$
\text { pris }
$$




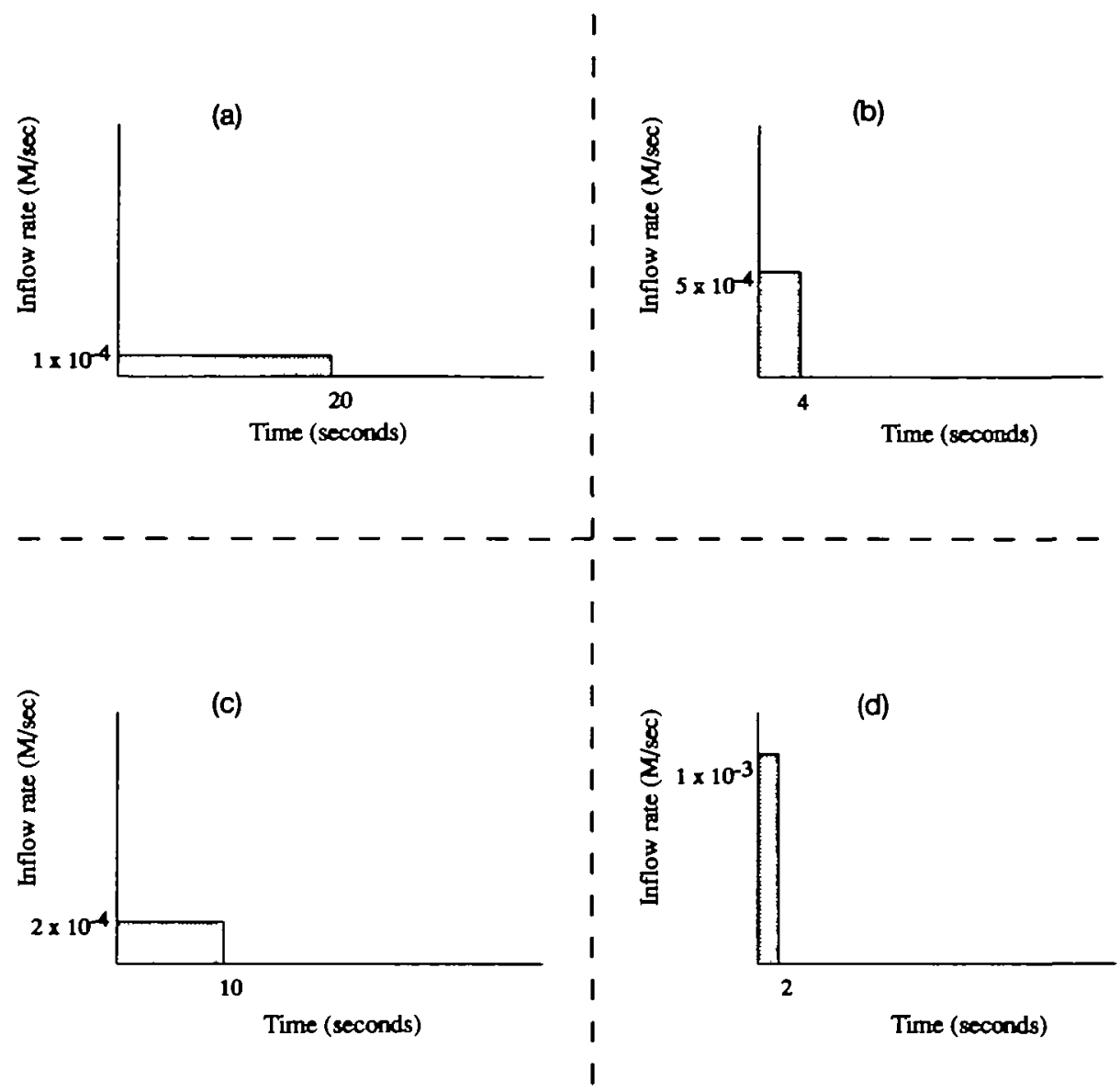

FIG. 6. Profiles of the boundary conditions used for the four experiments on influx rate: (a) flow rate $=0.0001 \mathrm{M} / \mathrm{sec}$ for 20 seconds; (b) flow rate $=0.0002 \mathrm{M} / \mathrm{sec}$ for $10 \mathrm{~seconds}$; (c) flow rate $=0.0005 \mathrm{M} / \mathrm{sec}$ for 4 seconds; and (d) flow rate $=0.001 \mathrm{M} / \mathrm{sec}$ for 2 seconds.

determine whether any factor associated with these changes other than the total amount of substrate injected has a significant effect on the final concentration profile of inactivated enzyme. For example, Fig. 6 shows several influx profiles where the total amount of substrate applied remains constant but the rate and duration of the process was varied. Figure 7 shows that the final inactive enzyme concentration profile, taken well after the finish of the application process, remained the same for the four different rates.

This is just one example of numerical experiments which may be done on generalized model systems to determine the dynamics of drug concentrations, in this case suicide substrates. By using the analytical solutions determined here, as numerical methods on more complex scenarios where analytical approaches are not possible, we believe that the dynamics of these substrate concentrations can be determined more accurately. 


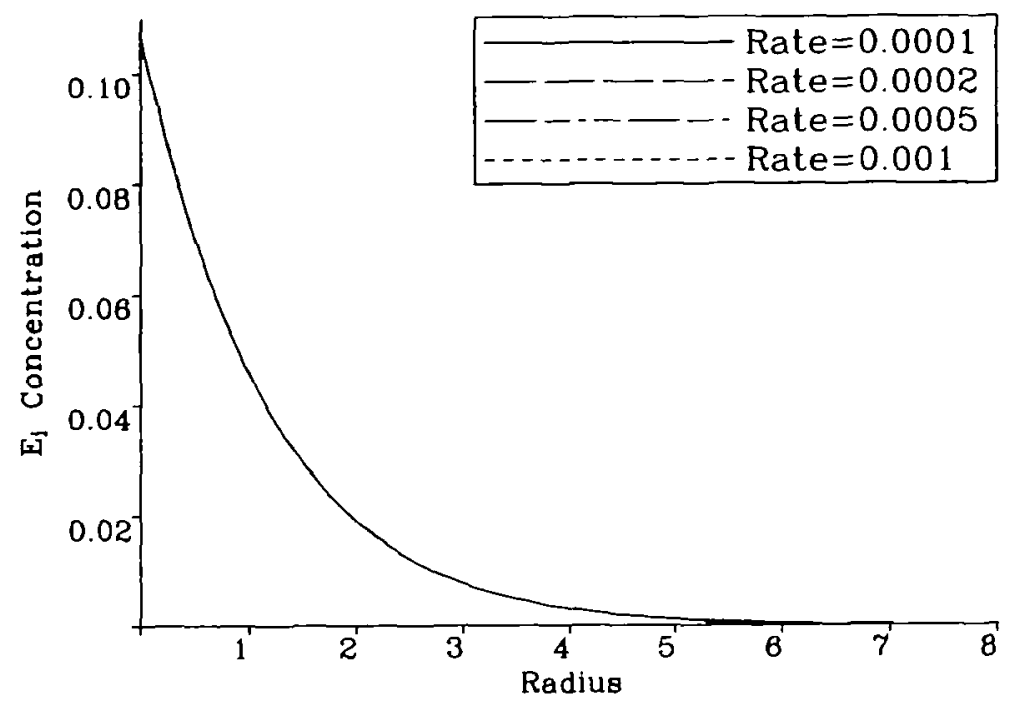

FIG. 7. Final-state inactive enzyme concentration profiles for each of the boundary conditions in Fig. 6. Parameters: $k_{1}=0.05, k_{-1}=1.0, k_{2}=1.0, k_{3}=1.0, k_{4}=1.0, E_{0}=1.0, D=0.005$.

\section{Acknowledgements}

MAB would like to acknowledge a Graduate Fellowship from the US National Science Foundation. This work (JDM) was in part supported by Grant DMS 90039 from the US National Science Foundation.

This article is based on a paper read at the Sixth IMA Conference on the Mathematical Theory of the Dynamics of Biological Systems, held in Oxford, 1-3 July 1992.

\section{REFERENCES}

Britton, N. F. 1991 An integral for a reaction-diffusion system. Appl. Math. Lett. 4, 43-7. Burke, M. A., Maini, P. K., \& Murray, J. D. 1990 On the kinetics of suicide substrates. Biophys. Chem., 37, 81-90.

Crank, J. 1975 The Mathematics of Diffusion. Oxford: Clarendon Press.

HeINEkEN, F., TsuchiYa, H., \& ARIS, R. 1967 On the mathematical status of the pseudo-steady state hypothesis of biochemical kinetics. Math. Biosci. 1, 95-113.

KeEner, J. P. 1988 Principles of Applied Mathematics, Transformation and Approximation. Reading, MA: Addison-Wesley.

MaINI, P. K., Burke, M. A., \& Murray, J. D. 1991 On the quasi-steady-state assumption applied to Michaelis-Menten and suicide substrate reactions with diffusion. Phil. Trans. R. Soc. Lond. A 337, 299-306.

MURRAY, J. D. 1977 Lectures on Nonlinear-Differential-Equation Models in Biology. Oxford: Clarendon Press.

Segel, L. A., \& Slemrod, M. 1989 The quasi-steady-state assumption: A case study in perturbation. SIAM Rev. 31, 446-77.

SEILER, N., JUNG, M. J., \& KOCH-WESER, J. (eds.) 1978 Enzyme-Activated Irreversible Inhibitors. Oxford: Elsevier Biomedical Press.

WALSH, C. T. 1984 Suicide substrates, mechanism-based enzyme inactivators: Recent developments. Ann. Rev. Biochem. 53, 493-535. 